\section{Die Funktion des Dopings}

\author{
Eine Technikfolgenabschätzung des \\ Spitzensports
}

\author{
von Swen Körner, Deutsche Sporthochschule \\ Köln
}

Im Spitzensport ist Enhancement ein altbekanntes Phänomen. Der Einsatz tatsächlich oder vermeintlich leistungsfördernder Substanzen und Verfahren ist hier seit langem verbreitet, in definierten Fällen verboten und wird dann als Doping sanktioniert. Der Beitrag stellt die Frage nach der Zukunft des Dopings im Spitzensport. Wenngleich die Zukunft vorlaufender Beobachtung per se entzogen bleibt, liefert eine systemtheoretisch informierte Analyse des Spitzensports Erwartbarkeiten: Die Erwartung einer auch künftig hohen Irritabilität des Systems durch Technologieentwicklungen, einer ungebrochenen Funktionalität von Doping, Dopingverbot und Dopingkontrolle sowie sich verschärfender Risiken der Risikobeobachtung. Neben dem Gendoping hängt das Doping der nächsten Gesellschaft an einer Technologieentwicklung der ganz anderen Art: dem Verbreitungsmedium Internet.

\section{Einleitung}

Stellten sich biomedizinische Herausforderungen bislang vorrangig als Suche nach einem ethisch verantwortlichen, sozial verträglichen und rechtlich umsetzbaren Umgang mit körperlichen und psychischen Krankheiten dar, so beziehen seit geraumer Zeit Möglichkeiten zum Enhancement gesunden Lebens gesellschaftsweit hohe Aufmerksamkeitswerte (Schöne-Seifert/Talbot 2009). Im Sport, und insbesondere im Spitzensport, auf den sich die folgenden Einlassungen konzentrieren, bezeichnet Enhancement in diesem Sinne ein altbekanntes Phänomen. Der Einsatz tatsächlich oder vermeintlich leistungsfördernder Substanzen und Verfahren ist hier seit Langem verbreitet, in definierten Fällen verboten und wird dann als Doping sanktioniert. In der Regel bildet der gedopte Athlet den „Zurechnungspunkt“ öffentlicher, medialer, rechtlicher, politischer, wissenschaftlicher oder pädagogischer Aufmerksamkei- ten. Im Unterschied zu schwer greifbaren Sozialstrukturen lassen sich Sportler sehr überzeugend zur Ursache des Dopingproblems stilisieren und in entsprechende Routinen gesellschaftlicher Culpabilisierung und Moralisierung überführen. Athleten aus Fleisch, Geist und Blut kann man aufsuchen, um Urin bitten, verurteilen oder Aufklärung zuteilwerden lassen. Strukturen nicht.

Im Abstand zum üblichen Empörungsgehalt einer Dopingdebatte, die notorisch Menschen braucht, um Doping zu begreifen, stellt der Beitrag in lockerer Anlehnung an das Paradigma der Technikfolgenabschätzung die Frage nach der Zukunft des Dopings im Spitzensport. Wenngleich die Zukunft vorlaufender Beobachtung per se entzogen bleibt (Kap. 2), liefert eine systemtheoretisch informierte Analyse des Spitzensports (Kap. 3) Erwartbarkeiten: Die Erwartung einer auch künftig hohen Irritabilität des Systems durch Technologieentwicklungen (Kap. 4 und 5), einer ungebrochenen Funktionalität von Doping, Dopingverbot und Dopingkontrolle sowie sich verschärfender Risiken der Risikobeobachtung (Kap. 6). Neben dem sog. Gendoping hängt das Doping der nächsten Gesellschaft an einer Technologieentwicklung der ganz anderen Art: dem Verbreitungsmedium Internet (Kap. 7).

\section{Technikfolgenabschätzung}

Technologieentwicklungen erzeugen Sicherheit, allerdings zum Preis neuer Unsicherheiten. Technikfolgenabschätzung (TA) begleitet den Bereich technologischer Entwicklung durch prospektive Analysen. Ungeachtet dessen, was daraus im Sinne gesellschaftlicher Orientierungs-, Steuerungs-, Kontroll- oder Prognosebedarfe abgeleitet wird, bietet TA Reflexion auf Zeitverhältnisse innerhalb zeitlicher Verhältnisse. Die in ihr entfaltete „Zukunft ist nichts außerhalb der Gegenwart" (Grunwald 2012, S. 145) - das gilt hinein bis in die letzte Nachkommastelle statistischer Risikokalkulationen. Das Wissen der TA ist Ergebnis gegenwärtiger Operationen, die Zukunft als gegenwärtige Zukunft einspielen, ,also im Modus einer fiktiv gesicherten (...) Realität" (Luhmann 1992, S. 187). Die zukünftige Gegenwart bleibt ungewiss. Insofern der Gegenstand der Beschreibung von seiner Beschreibung nicht 
abzulösen ist, sind Technikfolgen immer auch Folgen der Rede über Technikfolgen. Und Technikfolgenabschätzungen damit immer Teil des Problems, die einen eigenen Bereich des NichtWissens, der nicht-intendierten, nicht-antizipierten oder nicht-erwarteten Effekte erzeugen. ${ }^{1}$ Vorliegende Abschätzung zur Zukunft des Dopings im Spitzensport versteht ihren Beitrag in eben diesem reflexiven Sinne als „Ordnung mit Voraussicht" (Luhmann 2003, S. 57). Ihre Beschreibung ist gegenwärtige Beschreibung ${ }^{2}$ unter Ceteris-Paribus-Bedingungen (Grunwald 2010, S. 148), d. h. unter der Annahme einer prinzipiellen Strukturdeterminiertheit des beobachteten (wie auch des beobachtenden) Systems. Im System des Spitzensports geschieht, was geschieht - auf abschätzbarem Erwartungshorizont.

\section{Spitzensport der Gesellschaft}

Die moderne Gesellschaft erwartet ihre Zukunft spätestens seit dem 19. Jahrhundert als prinzipiell offen, gestaltbar und auf steten Zuwachs hin. Spitzensport ist eine ihrer Errungenschaften. Während Wissenschaft die Idee des Erkenntnisfortschritts radikalisiert, Politik das Programm staatlicher Wohlfahrt entwickelt, Wirtschaft vom merkantilistischen Summenkonstanzprinzip der Güter auf das Wachstumsparadigma umstellt und Erziehung den Menschen als ,steigerbare Realität" (Luhmann/Schorr 1979, S. 65) entdeckt, vollzieht sich im Sport und v. a. in Spitzensport die Übertragung des Prinzips Steigerung auf den menschlichen Körper (und Geist). Der moderne Spitzensport arrangiert den Vergleich von Leistung, v. a. von körperbasierter Leistung. Jemand wirft auf ein Tor, der andere sich dem Ball entgegen. Erst im Sportwettkampf wird derart Unwahrscheinliches in einer Weise wahrscheinlich, dass man nicht nur akut damit rechnen kann, sondern sich Lebensläufe zeitweise darauf einstellen sieht, in Leistungsvergleiche einzutreten, Positionen in Raum und Zeit auf physiologisch belastende und bisweilen hochriskante Weise zu verändern, Körper an Geräte zu schnallen, eintreffende Schläge zu akzeptieren, ein Zuspiel anzunehmen, auf einen Sprung einen eigenen folgen zu lassen oder im Pulk mit anderen über eine Linie zu laufen, an der man kurz vorher gestartet war. Sportwettkämpfe konditionieren in strenger Form, dass Leistungsmitteilungen auf Leistungsmitteilungen folgen, also Operationen gleichen Typs. Die Kontinuität des Systems liegt in der Diskontinuität seiner Ereignisse, und gerade nicht in der bloßen Ansammlung von Athleten, Trainern, Zuschauern, Stoppuhren, Schwingböden, Bällen, Schwimmhosen, Spikes, Arzneien oder Wurfgeräten.

Sportwettkämpfe preisen am Ende aus, was zu Beginn keinesfalls offensichtlich zu sein hat und deshalb artifiziell (durch Klassifikationssysteme, Start- und Ziellinien, die 0 auf Stoppuhr und Zentimetermeterband) auf „Null“ gebracht werden muss: den Unterschied von überlegener und unterlegener Leistung. Ausgehend vom Gebot prinzipiell gleich verteilter Chancen, das einen prinzipiell offenen Ausgang erwarten lässt, setzt Spitzensport eindeutige Unterschiede in die Welt, die sich in Vereinfachung hochkomplexer Vorgänge als überlegene oder unterlegene „Leistungen“ auf anwesende Körper, Personen oder Mannschaften zurechnen lassen und über Symbolik (Urkunden, Medaillen), Bilder (z. B. Zielfoto) und Zahlen (z. B. Tausendstelsekunde) zur Unterscheidung gebracht werden. Gegen Gleichheitsforderung und Quotenausgleich in Familie, Arbeitswelt, Wissenschaft und Politik, bringt der Spitzensport harte und anderweitig hoch umstrittene Unterschiede mit demonstrativer Geltung zur Ansicht.

\section{Steigerung durch Technologie}

Werden Leistungen gemessen, sind Vergleiche die Folge. Spitzensport setzt genau diese Vergleichslogik mit Steigerungserwartung in Serie. Bezeichnend hierfür ist die Aufzeichnung von Höchstleistungen. Rekorde ${ }^{3}$ machen in Raum und Zeit sowie sozial entfernte Leistungen aufeinander beziehbar. Als „geniale Abstraktionen“ ermöglichen sie den Vergleich von Damals und Heute, Lebenden und Toten und motivieren zur Anschlusshandlung (Überbietung). ${ }^{4}$

Aus dieser Steigerungserwartung folgt die strukturelle Technologiebetroffenheit des Spitzensports. Zum einen in Form von Technologien, die den Binnenraum des Menschen ansteuern (Biomechanik, Physiologie menschlicher Bewe- 
gung), während ein anderer Technologietyp außen ansetzt - um selbst dann noch Unterschiede und Steigerungen treffsicher abbilden zu können, wenn die Potenziale menschlicher Physiologie und Biomechanik ausgereizt sind (z. B. ultraschallgeschweißte Schwimmanzüge, ultraleichte Laufschuhe). Die selbst gesuchte Nähe des Spitzensports zur Technologie gründet in deren einfachen Versprechen, definierte Bereiche komplexer System-Umwelt-Beziehungen auszugrenzen, innerhalb derer wiederum definierte Elemente nach dem Schema von Ursache und Wirkung planmäßig gekoppelt werden können und somit Kontrolle, Steuerung und Prognose in Aussicht stellen. Das ist nicht nur die triviale Grundlage der Beziehung von Spitzensport zu Disziplinen angewandter Sportwissenschaft wie Sportmedizin, Trainingswissenschaft oder Sportpsychologie $^{5}$, sondern auch Basis seiner Dopingliäson: Erwartung und Versprechen „funktionierender Simplifikation im Medium der Kausalität" (Luhmann 2003, S. 97), eines gezielten An- und Ausschaltens leistungsrelevanter Parameter.

Doping stellt eine Art Knopfdrucktechnologie in Aussicht. Dopingtechnologien setzen dort an, wo der legitime Einfluss auf vermeintlich oder tatsächlich leistungslimitierende Körper- und Mentalprozesse an natürliche und oder moralische Grenzen stößt. ${ }^{6}$ Das „Gendoping“ bezeichnet die wohl zukunftsträchtigste Form anthropogener Leistungstechnologie, an der sich Risiken und Paradoxien des Dopings (wie auch des Redens darüber) besonders prägnant zeigen.

\section{Gendoping: Technologie zwischen Fakt und Fiktion}

Dient die Entwicklung von Verfahren und Substanzen zur zielgerichteten Beeinflussung der Genaktivität vorrangig der Identifikation neuer therapeutischer Strategien zur Behandlung von Krankheiten, so liegt die mögliche Nutzung der Ergebnisse biomedizinischer und pharmazeutischer Forschung zu Gendopingzwecken auf der Hand. Der Begriff „Gendoping“ wird in einem engeren und in einem weiten Sinn verwendet. Eng gefasst bezeichnet Gendoping das Einschleusen von genetischer Information (DNA oder RNA) in eine Zelle, ein Organ oder einen
Organismus durch gen- und zelltherapeutische Verfahren. In einem weiter gefassten Verständnis kann unter Gendoping auch eine gezielte Beeinflussung der Genaktivität durch andere Methoden verstanden werden (z. B. durch Einnahme/ Verabreichung pharmakologischer Wirkstoffe).

Entgegen populistisch aufgebauten „Freakshow"-Szenarien (Hungermann 2009) finden sich nach Gerlinger et al. (2008) gegenwärtig folgende mögliche Ansatzpunkte für Gendoping im Spitzensport: Gezielte Steuerung und Verbesserung der Energiebereitstellung ermöglichen u. a. Methoden zur Überexpression von Fettsäure- und Glucosetransportproteinen (FATP1, CD36, GLUTs), deren therapeutische Anwendung für die Behandlung von Adipositas- bzw. Diabetespatienten vorgesehen ist, aber gleichwohl insbesondere im Hochleistungssport als attraktives Verfügungswissen Einzug halten könnte. Entsprechende Verfahren befinden sich derzeit in der Phase präklinischer Erprobung. Weitere für spitzensportliche Zwecke hochinteressante Anwendungsfelder eröffnet der Bereich der Sauerstoffversorgung. Im Zentrum stehen hier seit der Isolierung des humanen EPO-Gens im Jahr 1983 insbesondere Strategien zur Erhöhung der Erythrozytenkonzentration und damit mittelbar der Sauerstoffaufnahme- sowie -transportkapazität. Zahlreiche Dopingfälle der letzten Jahre dokumentieren den Einsatz pharmakologisch hergestellter EPO-Präparate insbesondere in ausdauerintensiven Sportarten. Die nächste Entwicklungsstufe beinhaltet $u$. a. gentherapeutische Verfahren zur intramuskulären Applikation des EPO-Gens, die bereits im Zusammenhang von Ermittlungen gegen einen bekannten ehemaligen Leichtathletik-Bundestrainer aufgetaucht sind (unter dem Markennamen Repoxygen ${ }^{\mathrm{TM}}$ des Unternehmens Oxford BioMedica). Der Skelettmuskelaufbau bildet schließlich die dritte molekulare Zielgröße, die gegenwärtig für Gendopingzwecke nutzbar gemacht werden kann. Neben dem Einsatz gentechnisch hergestellter Wachstumshormone wie HGH (Human Growth Hormone) und IGF-1 (Insulin like Growth Factors), für den nach aktuellem Stand der Analytik valide Nachweismöglichkeiten fehlen (Franke 2007), verdienen v. a. gezielte Strategien zur Überexpression des Rezeptorproteins PPAR-del- 
ta sowie zur Blockade des extrazellulären Botenstoffs Myostatin besondere Beachtung. Sowohl die Umwandlung von Muskelfasern des Typs II (schnelle Fasern) in TYP I-Fasern (langsame Fasern) durch Modulation des PPAR-delta Rezeptors als auch die Hemmung des Myostatin-Gens mittels z. B. inhibierender RNA, die eine Erhöhung sowohl des Muskelquerschnittwachstums als auch der Hyperplasie (Erhöhung der Faseranzahl) bewirkt, konnte bereits im Tierversuch nachgewiesen ${ }^{7}$ und $z$. T. in klinische Studien überführt werden.

In der Vorstellung einer gezielten Aktivierung und Steuerung leistungsrelevanter Parameter durch Doping und Gendoping steckt freilich eine Portion Mythos. Technologische Eingriffe in komplexe Systeme, und zu ihnen zählt der einzelne menschliche Organismus, haben mit Nicht-Linearitäten zu rechnen, mit nicht-intendierten Effekten, die dann wiederum aufwendig durch weiteren Technologieeinsatz im Sinne eines Containments beherrschbar gemacht werden müssen. (Genetisches) Doping ist eine riskante Technologie, eine Simplifikation im Medium der Kausalität, die angesichts der Komplexität sportlichen Erfolgs regelmäßig an die Grenze des Simplifizierbaren stößt (Lames 2002). So ist auch beim Einsatz von Verfahren und Substanzen zur Modifikation der Genaktivität mit dem Ziel der Leistungssteigerung bei gesunden Menschen zurzeit von schwer kalkulierbaren gesundheitlichen Risiken auszugehen. Bekannte Nebenwirkungen (wie z. B. Immunreaktionen oder unkontrolliertes Zellwachstum) weisen auf potenziell massive gesundheitliche Schäden hin, die letztendlich auch zum Tod führen können (Beiter/Velders 2012). Entwicklungen im Feld gentechnologischer Verfahren sind von einer erkennbar hohen Dynamik gekennzeichnet. Zugleich ist mit Blick auf systemtypische Erwartungsstrukturen von einer eher niedrigen Missbrauchsschwelle in Anwendungskontexten sportlicher Leistungssteigerung auszugehen. Entsprechend hat die Welt-Anti-Doping-Agentur (WADA) im Jahr 2003 Gendoping in ihren AntiDoping-Code aufgenommen.

Während Medizin und Molekularbiologie das aktuelle und zukünftige Potenzial ebenso wie die Risiken des notwendig an Erfolge gentherapeutischer Forschung gekoppelten Gendopings im Leistungssport unterschiedlich einschätzen (Beiter/Velders 2012; Diel/Friedel 2007), tragen insbesondere Resonanzen im öffentlich-medialen Raum regelmäßig Züge eines Krisendiskurses, in dem entgegen aller fachlich begründeten Einwände und Unsicherheiten der geklonte Athlet als reale Utopie des Spitzensports von Morgen heraufbeschworen wird (Körner/Schardien 2012).

\section{Risiken und Paradoxien}

Aus Sicht der Systemtheorie bezeichnet Doping zunächst ein Umweltproblem, d. h. ein sozial nicht direkt handhabbares Geschehen in der psychischen und biologischen Umwelt der Gesellschaft. ${ }^{8}$ Um diese Umwelt herum ist eine ganze Armada von Symbolen und Strukturen entstanden, die Imaginationen von Erreichbarkeit und Steuerbarkeit erzeugen. Dazu zählt neben betreuender Medizin, Physiotherapie, Ernährungsberatung, Psychologie, Trainingslehre schließlich auch das moderne Dopingkontrollwesen.

Mit dem Kontrollwesen hat sich eine Sonderform von „Beobachtung zweiter Ordnung“ institutionell auf Dauer gestellt. Ist die überlegene/unterlegene Leistung auch eine saubere? Der Wettkampf selbst gibt keine Garantie. Vielmehr ist eine enorm ressourcenaufwändige ${ }^{9}$ soziale Operation (Urin- bzw. Blutanalyse) von Nöten, um Klarheit in undurchsichtige Körperverhältnisse zu bringen und eine mehr oder weniger glaubwürdige Gegensymbolik zur de facto fehlenden Steuerbarkeit zu installieren. Das Dopingkontrollwesen bedient sich dazu besonderer Verfahren (Melde- und Kontrollsysteme ${ }^{10}$ ) und Symbole (A- und B-Probe), die möglichst eindeutige Informationen (gedopt/nicht gedopt) über die Körperumwelt (Blut, Urin) gewinnen, um damit das Vertrauen in das System des Leistungsvergleichs zu stabilisieren oder (wieder-) herzustellen. Für den Spitzensport selbst handelt es sich demnach um eine hochfunktionale Einrichtung (Emrich/Pitsch 2009). Ist die Probe positiv, stabilisiert das Testergebnis das Vertrauen in die Wirksamkeit des Kontrollwesens, die Beherrschbarkeit des Problems und nicht zuletzt darin, dass es vom organisierten Sport 
hinreichend ernst genommen wird. Zugleich ermöglichen überführte Körpersäfte den Mechanismus der Schuldabwälzung. Als Authentizitätsmarker lenken sie den Blick auf einzelne Dopingsünder, die mit regelmäßig vernehmbarer Empörung ausgeworfen werden, während der Betrieb ungestört weiterlaufen kann. ${ }^{11}$ Dem gegenüber stabilisiert jede negative Probe offensichtlich die Moral des Sports, d. h. die Erwartung in einen sauberen, d. h. noch fairen, noch natürlichen und noch gesunden (d. h. noch menschlichen) Spitzensport.

Die Grenze zwischen ,gedopt“ und „,nichtgedopt" ist eine Sinngrenze und verläuft auf schmalem Grat. Das zeigt ein Blick auf den Code der Welt-Anti-Doping Agentur, auf Grenzwertdiskussionen und auf Möglichkeiten, die gerade aus dem Bereich der Gentechnologie auf sich aufmerksam machen. Eine selten beachtete Paradoxie des Dopingkontrollsystems liegt darin, im Bemühen um die Überwachung der Einhaltung natürlicher (oder gesunder oder fairer) Grenzen jeweils mit anzugeben, wie sehr hier Natur (Gesundheit, Fairness) nur als gleitende soziokulturelle Grenzziehung, etwa durch biostatistisch ermittelte Normbereiche und damit auch als anders möglich, zu haben sind. ${ }^{12}$ Unterschiede, die es eigentlich zu hypostasieren gilt, werden durch eigene Beobachtung als Unterscheidungen sichtbar, d. h. als Zwei-Seiten-Formen, und damit dem Kosmos der Essenzen enthoben. Wer Natur bezeichnet, der tut dies immer schon im Rahmen einer Unterscheidung, die Kultur erzeugt, auch wenn man auf die Gegenseite kreuzt. Das Kontrollwesen jedenfalls zieht die es tragenden Grenzen selbst, bisweilen verschiebt es sie. Und auch seine Evidenzproduktion in Form eindeutiger A- und B-Proben erscheint letztlich nur solange plausibel, wie die Verfahren der Erzeugung im toten Winkel verbleiben (Pitsch 2009). Zu den erwartbaren, aber wohl kaum intendierten Nebenfolgen der Anti-Dopingforschung gehört zudem, am Fortschritt genau jener Entwicklung mitzuwirken, zu deren Begrenzung sie antritt. Das von ihr erzeugte Wissen um Wirkungsweisen bestimmter Dopingverfahren und Substanzen präzisiert nolens volens das Wissen für deren Nutzbarmachung im Dopingkontext.

\section{Das Doping der nächsten Gesellschaft}

Der moderne Spitzensport definiert sein Spiel konsequent entlang einer doppelten Erwartung: der Erwartung von Höchstleistung bei gleichzeitig vorzeigbarer Moral. Dass Leistungen nicht sinken, sondern tendenziell steigen, dafür sorgt der hochselektive Zugriff auf die Körper und Psychen von Athleten sowie die strukturelle Kopplung an eine betreuende Sportwissenschaft bzw. Technologieentwicklung. Und dass die Moralerwartung dabei konstant gehalten werden kann, ist die Leistung verbindlicher Regelwerke, die den Spielraum möglicher Verhaltensselektionen einschränken. Doping wie auch sein in den 1960-er Jahren eingesetztes Verbot dienen in dieser Optik der Kompensation vom Spitzensport selbst erzeugter Effekte. Während Doping auf innovative Weise die Seite der technologischen Steigerungserwartung im Spitzensport bedient, setzt das Dopingverbot die in ihm strukturell eingebaute Steigerungs- und Rekordlogik zwischen die Leitplanken einer großen Moral, die mehr erwarten lässt als bloße Treue zur Spielregel: Du sollst (höchst-)leisten, aber mit reinem Herzen, d. h. nicht dopen. Überführte Doper gelten einem verbreiteten Sprachgebrauch zu Folge als „Doping-Sünder" - die implizit-argumentative Metaphorik verweist auf die Tiefe der Schuldanklage. Doping ist kein zufälliger Missgriff, den man mit einem saloppen je ne sais pas aus der Eigenzurechnung katapultieren kann. Doping wird verhandelt als Frage substanzieller Eigenschaften, auf die ein Sportcharakter schattenhaft festgelegt wird. Entscheidend ist, dass sich der Spitzensport auf beiden Seiten der Unterscheidung bewegt, er seine gesellschaftliche Reproduktion als Einheit der Differenz von Konformität und Abweichung, Sauberkeit und Nichtsauberkeit vollzieht - so unbefriedigend das für moralische Beobachter sein mag. ${ }^{13}$ Insofern erscheinen jene sozial eingeübten Beschreibungen überdenkenswert, die am Spitzensport eigene Bedürfnislagen, Erwartungen und Nutzeninteressen (Stichwort: Gesundheit, Integration, Vorbildfunktion) hartnäckig als die seinen ausgeben.

Doping ist gegenwärtig und wohl auch künftig nicht nur im Spitzensport funktional. Eine Vielzahl weiterer gesellschaftlicher Beobachter 
entwickelt aus dieser Form abweichenden Verhaltens Dynamik. Offensichtlich stellen weder ethische, rechtliche, mediale noch pädagogische Beobachter ihren Betrieb mit Blick entdeckte Doper ein. Und so wie die mit guten Absichten ausgestattete Präventionsarbeit am noch-nicht mündigen Athleten am und mit Doping Zukunft in eigener Sache erwirtschaftet, prozessiert auch das wissenschaftliche Kontrollwesen in einer Art Kybernetik des Heuschreckenflugs (Luhmann 1983): Einmal gestartet, reagiert man letztlich auf selbst erzeugte Turbulenzen. ${ }^{14}$ Je intensiver Kontrolle, desto subtiler die Abweichung, desto mehr Kontrollbedarf usw.

Für die Zukunft des Dopings bislang wenig beachtet ist die Rolle des Internets. Mit der Durchsetzung neuer Verbreitungstechnologien verlieren angestammte Orte und Autoritäten (Priester, Kirchen, Universitäten, Gelehrte) ihr Wissens- und Wissensproduktionsmonopol. Wie der Buchdruck im 15. Jahrhundert, bereitet gegenwärtig das Internet den Übergang zur nächsten Gesellschaft (Baecker 2007). Die prinzipiell ort- sowie zeitunabhängige Zugriffsoption auf eine unbestimmte Vielfalt kultureller Inhalte, die Möglichkeit echtzeitbasierter Kommunikation sowie die Auflösung herkömmlicher Rollenasymmetrien (Laie vs. Experte) machen das Web $2.0 \mathrm{zu}$ einem einflussreichen Medium für die Produktion, Transformation und Aneignung von Wissen aller Art. Wer heute dopen will, ist längst nicht mehr angewiesen auf Rat und Tat seines Arztes oder Apothekers. Erzeugung dopingrelevanten Wissens und Distribution entsprechender Technologien verlaufen polyzentrisch und kollateral, darin wesentlich begünstigt durch das World Wide Web. Dem Medium Internet und dem Phänomen Doping ist eine netzwerkartige Struktur gemein. Doping vollzieht sich auf den Hinterbühnen des Sports, legitimiert von einer eingespielten Untergrundmoral. Das Internet eröffnet dafür einen strukturell günstigen Interaktionsraum: Die vermeintlich anonyme Abgeschlossenheit sozialer Netzwerke ermöglicht eine selbstläufige, von wissenschaftlicher, politischer und öffentlicher Kontrolle weitgehend entzogene Rezeption, Diskussion, Bewertung und Aneignung vermeintlich oder tatsächlich leistungssteigernder Dopingpraktiken. ${ }^{15}$ Die Schubwirkung für das Doping der nächsten Gesellschaft dürfte beträchtlich sein.

\section{Anmerkungen}

1) $\mathrm{Zu}$ dieser Folgenunterscheidung siehe Merton 1998.

2) Und sie ist: Beschreibung, d. h. selbstreferentielle Erzeugung von Fremdreferenz, also letztlich Verdopplung ohne Original (Nassehi 2010, S. 204ff.).

3) Anfangs als Begriff für die Aufzeichnung von Leistung (engl. to record), später dann als Begriff für Höchstleistung selbst.

4) $\mathrm{Zu}$ den ,genialen Abstraktionen" siehe Guttman 1979, S. 59. Radrennfahren, 100m-Lauf und Stabhochsprung z. B. verzeichnen seit den ersten modernen Olympischen Spielen im Jahr 1896 Leistungssteigerungen von 24-221 Prozent (s. das Editorial in Nature Materials 2012, S. 651).

5) Politisch lanciert in den späten 60er-Jahren des 20. Jahrhunderts vor dem Hintergrund der Olympischen Spiele 1972 in München. Die sportwissenschaftliche Programmierung auf Anwenderinteressen des modernen Leistungssports ist seither dominant (DVS/DGSP/DSB 2005).

6) Der Körper als organisch-physische Materialitätsbasis sportlicher Leistung setzt Grenzen der Machbarkeit. Z. B. nähert sich die Proteinumsatzrate unter Belastung des zellulären Systems einem Wachstumsplateau. Natur bezeichnet hier eine empirische Grenznorm biologischer Adaptation. Natur ist aber auch als moralisches Argument für Nicht-Machbarkeit im Einsatz. Beispielsweise dann, wenn in Folge des Einsatzes von im weitesten Sinne Technologie die Einhaltung ,menschlicher" Grenzen angemahnt wird, etwa im Kontext von (Gen-)Doping. Über die Natur des Menschen, so heißt es dann in strenger Geste, dürfe so nicht verfügt werden; eine Forderung, die freilich den Preis der Paradoxie zahlt, insofern sie selbst über etwas verfügt, was sich nach eigener Aussage eigentlich der Verfügung entziehen soll (Nassehi 2003).

7) $\mathrm{Zu}$ den „Marathonmäusen“ und den „Knockout"-Mäusen siehe Ferrari et al. 2010.

8) Verstanden als heimlicher Griff zur Pille oder Spritze sowie körperliche Folgezustände, nicht als Thema der Kommunikation.

9) Die jährlichen weltweiten Ausgaben für Dopingtests liegen aktuell im Schnitt bei über 300 Millionen Dollar. 0,3 Prozent der Tests führen letztlich zu einer Sperre. 
10) Körper (und Psychen) müssen erreichbar sein, mit allem, was dazu gehört, z. B. dem seit 2009 eingesetzten Online-Meldesystem ADAMS der WADA.

11) Für den organisierten Sport ist Doping insofern „brauchbare Illegalität“" (Luhmann 1976, S. 304).

12) Zur Problematik gängiger Verbotskriterien vgl. Gugutzer 2009.

13) Angaben zu aktuellen Prävalenzraten von Doping im deutschen Spitzensport variieren zwischen sechs Prozent (Breuer/Hallmann 2012) und 35 Prozent (Pitsch et al. 2009).

14) Für derartige Selbstbezüglichkeiten innerhalb der (wissenschaftlichen) Krisenkommunikation über „dicke Kinder“"vgl. Körner 2008.

15) Das Zentrum für präventive Dopingforschung der Deutschen Sporthochschule Köln betreibt u. a. ein Monitoring des weltweiten Marktes online verfügbarer Dopingpräparate sowie einschlägiger Diskussions- und Austauschforen von Dopingusern im Internet.

\section{Literatur}

Baecker, D., 2007: Studien zur nächsten Gesellschaft. Frankfurt a. M.

Beiter, T.; Velders, M., 2012: Pimp My Genes - Gendoping zwischen Fakt und Fiktionen. In: Deutsche Zeitschrift für Sportmedizin 63/5 (2012), S. 16-26

Breuer, C.; Hallmann, K., 2013: Dysfunktionen des Spitzensports: Doping, Match-Fixing und Gesundheitsgefährdungen aus Sicht von Bevölkerung und Athleten. Deutsche Sporthilfe

Diel, P.; Friedel, U., 2007: Gendoping: Techniken, potenzielle biologische Ziele und Möglichkeiten des Nachweises. Deutsche Sporthochschule Köln

DVS - Deutsche Vereinigung für Sportwissenschaft; DGSP - Deutsche Gesellschaft für Sportmedizin und Prävention; DSB - Deutscher Sportbund, (2005): Memorandum zur Entwicklung der Sportwissenschaft Emrich, E.; Pitsch, W., 2009: Zum Dopingkontrollmarkt - Sind Investitionen in den Anschein von Ehrlichkeit lohnender als die Ehrlichkeit selbst? In: Emrich, E.; Pitsch, W. (Hg.): Sport und Doping. Zur Analyse einer antagonistischen Symbiose. Frankfurt a. M., S. 111-130

Ferrari, A.; Coenen, Chr.; Grunwald, A. et al., 2010: Animal Enhancement. Neue technische Möglichkeiten und ethische Fragen. Bern

Franke, E., 2007: Doping - Ein nicht zufälliges Dilemma: Die (traditionelle) Athletenverantwortung in der (globalen) Systemwelt des Sports. Berlin
Gerlinger, K.; Petermann, T.; Sauter, A. (Hg.), 2008: Gendoping. Wissenschaftliche Grundlagen - Einfallstore - Kontrolle. Studien des Büros für Technikfolgen-Abschätzung beim Deutschen Bundestag, Bd. 28. Berlin

Grunwald, A., 2010: Technikfolgenabschätzung Eine Einführung. Berlin

Gugutzer, R., 2009: Doping im Spitzensport der reflexiven Moderne. In: Sport und Gesellschaft 6/1 (2009), S. 3-29

Guttmann, A., 1979: Vom Ritual zum Rekord. Das Wesen des modernen Sports. Schorndorf

Hungermann, J., 2009: Vorbild oder Freakshow? Der Spitzensport steckt in der Krise. In: Welt Online (4.8.2009); http://www.welt.de/welt_print/debatte/ article4252242/Vorbild-oder-Freakshow.html (download 3.5.13)

Körner, S., 2008: Dicke Kinder - Revisited. Zur Kommunikation juveniler Körperkrisen. Bielefeld

Körner, S.; Schardien, S., 2012: Höher - schneller weiter. Gentechnologisches Enhancement im Spitzensport. Ethische, rechtliche und soziale Perspektivierungen. Paderborn

Lames, M., 2002: Leistungsentwicklung in der Leichtathletik - ist Doping als leistungsfördernder Effekt identifizierbar? In: dvs-Informationen 17/4 (2002), S. $15-22$

Luhmann, N., 1976: Funktionen und Folgen formaler Organisationen. Berlin

Luhmann, N., 1983: Anspruchsinflation im Krankheitssystem. Eine Stellungnahme aus gesellschaftstheoretischer Sicht. In: Herde-Dornreich, P.; Schuller, A. (Hg.): Die Anspruchsspirale: Schicksal oder Systemdefekt? Stuttgart, S. 28-49

Luhmann, N., 1992: Beobachtungen der Moderne. Opladen

Luhmann, N., 2003: Soziologie des Risikos. Berlin

Luhmann, N.; Schorr, K.E., 1979: Reflexionsprobleme im Erziehungssystem. Stuttgart

Merton, R.K., 1998: Unanticipated Consequences and Kindred Sociological Ideas: A Personal Gloss. In: Mongardini, C.; Tabboni, S. (Hg.): Robert K. Merton and Contemporary Sociology. New Brunswick, S. 295-318

Nassehi, A., 2003: Geschlossenheit und Offenheit. Studien zur Theorie der modernen Gesellschaft. Frankfurt a. M.

Nassehi, A., 2010: Mit dem Taxi durch die Gesellschaft. Soziologische Storys. Hamburg

Nature Materials, 2012: More Than Training. Editoral. In: Nature Materials 11/8 (2012), S. 651 
Pitsch, W., 2009: Dopingkontrollen zwischen Testtheorie und Moral - Nicht intendierte Folgen prinzipiell nicht perfekter Dopingtests. In: Emrich, E.; Pitsch, W. (Hg.): Sport und Doping. Zur Analyse einer antagonistischen Symbiose. Frankfurt a. M., S. 95-109

Pitsch, W.; Maats, P.; Emrich, E., 2009: Zur Häufigkeit des Dopings im deutschen Spitzensport - eine Replikationsstudie. In: Emrich, E.; Pitsch, W. (Hg.): Sport und Doping. Zur Analyse einer antagonistischen Symbiose. Frankfurt a. M., S. 19-36

Schöne-Seifert, B.; Talbot, D. (Hg.), 2009: Enhancement. Die ethische Debatte. Paderborn

Stichweh, R., 2005: Der Wettkampfsport und sein Publikum: Risikoverhalten und Selbstbegrenzung im modernen Hochleistungssport. In: Stichweh, R.: Inklusion und Exklusion. Studien zur Gesellschaftstheorie. Bielefeld, S. 113-129

\section{Kontakt}

Prof. Dr. Swen Körner

Institut für Pädagogik und Philosophie

Deutsche Sporthochschule Köln

Am Sportpark Müngersdorf 6, 50933 Köln

Tel.: +49 (0) 221 / 4982 - 3810

E-Mail: Koerner@dshs-koeln.de

\section{$《 》$}

\section{ITAS-Newsletter}

Mit dem online verfügbaren ITAS-Newsletter informiert das Institut für Technikfolgenabschätzung und Systemanalyse (ITAS) über Projekte, neue Publikationen, Personalia und kommende Veranstaltungen des Instituts. Der Newsletter bündelt und komprimiert für einen Zeitraum von etwa vier bis sechs Wochen die Neuigkeiten, die zuvor sukzessive im Internetangebot des Instituts angezeigt wurden. Vom Online-Newsletter führen Links direkt zu den ausführlicheren Informationen auf dem ITAS-Server. Damit erhält der interessierte Nutzer über das sich laufend erweiternde Serverangebot ein zeitnahes Informationsangebot. Für den Vertrieb des ITAS-Newsletters wird ein Dienst des Deutschen Forschungsnetzes verwendet. Anmeldungen sind möglich unter http://www.itas.kit.edu/ newsletter.php. Bei Fragen und auftretenden technischen Problemen schicken Sie bitte eine E-Mail an itas-newsletter-request@listserv.dfn.de.

\section{Visionen von Animal Enhance- ment und Perspektiven für die Technikfolgenabschätzung}

\author{
von Arianna Ferrari, ITAS
}

Obwohl der zeitgenössische Diskurs um „Enhancement" sich in besonderer Weise lange auf menschliche Fähigkeiten bezog, war schon zu Beginn klar, dass die Rede einer Optimierung biologischer Merkmale sich auch auf andere Lebewesen erstrecken kann. Visionen der Verbesserung dessen, was je nach Bereich als „nützliche“ oder „wichtige“" Eigenschaft eines Tieres gewertet wird, prägen die heutige experimentelle Forschung - sowohl im landwirtschaftlichen und biomedizinischen Bereich als auch bei Anwendungen in Sport und Heimtierhaltung. Durch die Entwicklung neuer Technologien verändert sich die Mensch-Tier-Beziehung, da zum Teil neue Bedürfnisse geweckt werden und neue Kontroversen ausgelöst werden, die auch dazu dienen können, bereits etablierte Nutzungen in Frage zu stellen. Damit erlangen Visionen von Animal Enhancement auch eine Bedeutung für die TA.

\section{Was bedeutet Animal Enhancement?}

Obwohl sich der zeitgenössische Diskurs um „Enhancement" (Verbesserung, Optimierung) in Bezug auf menschliche Fähigkeiten entwickelt hat (Roco/Bainbridge 2002) und das zum größten Teil auch immer noch tut, war von Anfang an klar, dass die Rede einer Optimierung biologischer Merkmale nicht gattungsspezifisch ist und sich auch auf alle Lebewesen erstrecken kann. So wie der Mensch ,verbessert“" werden kann bzw. seine Fähigkeiten neu gestalten und besser an gewünschten Zielen angepasst werden können, so können auch Eigenschaften von Tieren verbessert werden (Ferrari et al. 2010). Der Begriff ,Enhancement" kann durch die Formel ,jemand verbessert etwas unter einem Kriterium“" sprachpragmatisch rekonstruiert werden, da es bei solchen Handlungen immer Akteure gibt, die etwas gemäß bestimmter Kriterien verbessern (Grunwald 2008). Darüber hinaus hat der Begriff eine starke positive Konnotation, woraus er seine rhetorische Kraft entwickelt. 